\title{
INHERITED SMALLPOX
}

BY

J. PICKFORD MARSDEN, M.A., M.D.(Cantab.), D.P.H., Deputy Medical Superintendent,

AND

C. R. M. GREENFIELD, M.B., B.S.(Lond.), Assistant Medical Officer, River Hospitals, London County Council.

Most authorities record that the association of pregnancy and smallpox is malign and that abortion, due to uterine haemorrhage, frequently results from it. At the same time it is recognized that inherited infection is commoner in smallpox than in any other acute illness and many references are to be found in the literature to this subject of smallpox in pregnancy and its effect upon the foetus. Schamberg and Kolmer ${ }^{1}$ discuss inherited infection and record a few cases. The generally accepted view appears to be that this type of infection is fortuitous and that there is no fixed relation between the onset of the disease as it occurs in the mother and as it may occur in the child. From the literature it is to be gathered that in the later stages of pregnancy the child may be born alive or dead, suffering from smallpox, incubating the disease or having recovered from it in utero. But we have altogether failed to find any favourable reference to time-incidence of attacks as a factor of importance in maternal and foetal smallpox; or, for example, any set of data which would suggest that the incidence of inherited infection may vary in accordance with the stage which the pregnancy has reached when the woman contracts smallpox, or that it may bear some relation to the time-interval between the onset of her attack and the birth of the child.

In an attempt to obtain some more definite information on this aspect of the subject, we have recently analyzed the records of relevant cases which have been under our care in the London smallpox hospitals during the last six years. We have watched many women, at all stages of pregnancy, preserve that state through an attack of smallpox and we have encountered only two who aborted. But we wish to emphasize that the fact that throughout this period all save fourteen patients, none of whom was pregnant, out of a total of over thirteen thousand admitted to hospital, suffered from the results of infection with a benign strain of virus and consequently from modified smallpox, very materially enhanced our opportunities for observation. 


\section{Case records.*}

Our cases, thirty-four in number, fall into four groups :-

Group 1.-True congenital smallpox.

Case 1.-Onset of mother's illness 20.6.29; rash 22.6.29. Child born 29.6.29, ' rash present at birth '; admitted to hospital 1.7.29; smallpox rash then vesiculopustular (about third day); died 2.7.29.

Case 2.-Onset of mother's illness 20.4.31; rash 22.4.31. Child born 29.4.31 (? premature) ' rash present at birth'; admitted to hospital 6.5.31, smallpox rash then 8 or 9 days old; recovered.

Case 3.-Onset of mother's illness 22.2.32; rash 25.2.32. Child born in hospital 6.3.32 with smallpox rash present estimated to be 3 or 4 days old; recovered.

These three cases were born alive at or about full term, and in each case a period of about ten days elapsed between the onset of the mother's illness and the estimated time of appearance of the exanthem on the child. The significance which we attach to this latter point will be referred to in the general discussion.

Group 2.-Inherited infections (type ' $A$ ') •

Case 4.-Onset of mother's illness 16.2.29; rash 19.2.29. Child born 16.2.29; vaccinated successfully 22.2.29; admitted to hospital 28.2.29; rash appeared 26.2.29, on eleventh day of extra-uterine life and of mother's illness; recovered.

Case 5.-Onset of mother's illness 21.1.30; rash 23.1.30. Child born 21.1.30; admitted to hospital and vaccinated successfully 27.1.30; rash 31.1.30 on eleventh day of extra-uterine life and of mother's illness; recovered.

Case 6.-Onset of mother's illness 26.4.30; rash 29.4.30. Child born 26.4.30; admitted to hospital and vaccinated successfully 1.5 .30 ; rash 5.5.30 on tenth day of extra-uterine life and of mother's illness; recovered.

Case 7.-Onset of mother's illness 27.1.30; rash 29.1.30. Child born 28.1.30; admitted to hospital and vaccinated successfully 31.1.30; rash 8.2.30 on twelfth day of extra-uterine life and thirteenth day of mother's illness : recovered.

Case 8.-Onset of mother's illness 25.4.31; rash 26.4.31. Child born in hospital and vaccinated successfully 30.4.31; rash 7.5.31 on eighth day of extra-uterine life and thirteenth day of mother's illness; recovered.

Case 9.-Onset of mother's illness ? 25.1.34; rash 29.1.34. Child born 25.1.34; rash 3.2.34, i.e., tenth day of extra-uterine life and of mother's illness; recovered.

Case 10.- Onset of mother's illness 7.6.31; rash 8.6.31. Child born in hospital and vaccinated successfully 14.6.31; rash 19.6.31, i.e., sixth day of extra-uterine life and thirteenth day of mother's illness; recovered.

Case 11.-Onset of mother's illness 2.11.30; rash 4.11.30. Binovular twins born in hospital and vaccinated successfully 9.11.30; rash appeared on each twin 14.11.30, i.e., sixth day of extra-uterine life and thirteenth day of mother's illness; recovered.

In this group infection was acquired in utero, or possibly during separation from the parent, and in each case the child's rash appeared within fourteen days of birth. In each case the mother at the time of parturition was either in the pre-eruptive stage of smallpox or in the early days of efflorescence. The common interval of nine to twelve days between the onset of the mother's toxaemia and the appearance of the child's rash is again to be remarked.

* For permission to publish clinical details of these cases we are indebted to Dr. A. F. Cameron, Medical Superintendent of the River Hospitals. 
Group 3.-Inherited infections (type ' $B$ ').

Case 12.-Onset of mother's illness 12.2.30; rash 14.2.30. Child born (? premature) 16.2.30; admitted to hospital and vaccinated successfully 19.2.30; rash 26.2 .30 , i.e., eleventh day of extra-uterine life and fifteenth day of mother's illness; died.

Case 13.-Onset of mother's illness 29.3.30; rash 31.3.30. Child (first of binovular twins) born in hospital and vaccinated successfully 6.4.30; rash 16.4.30, i.e., eleventh of extra-uterine life and nineteenth day of mother's illness; recovered. The second (case 25) did not develop smallpox.

Case 14.-Onset of mother's illness 5.6.30; rash 7.6.30. Child born in hospital and vaccinated successfully 7.6 .30 ; rash 18.6 .30 , i.e., twelfth day of extra-uterine life and fourteenth day of mother's illness; recovered.

Case 15. - Mother successfully vaccinated 24.5.30; rash 4.6.30. Child born 4.6.30; admitted to hospital and vaccinated successfully 6.6.30; rash 14.6.30, i.e., eleventh day of extra-uterine life and thirteenth day of mother's illness; recovered.

Case 16.-Onset of mother's illness 30.7.30; rash 1.8.30. Child born 1.8.30; admitted to hospital and successfully vaccinated 4.8.30; rash 12.8.30, i.e., twelfth day of extra-uterine life and fourteenth day of mother's illness.

Case 17.-Mother vaccinated successfully 8.12.30; rash 9.12.30. Child born 10.12.30; admitted to hospital and vaccinated successfully 13.12.30; rash 21.12.30, i.e., on twelfth day of extra-uterine life and fifteenth day of mother's illness.

The common factors in this group are the appearance of the focal rash in each case on the eleventh or twelfth day of life, and the fact that in each case (with one exception) the child was born actually at the time of outcrop of the mother's focal rash. During the delivery of case 13 the mother's rash was six days old.

Group 4.-Infants born of infected mothers, but who escaped congenital infection.

Some of these acquired smallpox subsequent to birth.

A. Born IMMEdiATELY PRIOR TO THE ONSET OF THE MOTHER'S ILLNESS.

Case 18.-Onset of mother's illness ? 9.1.31; rash 11.1.31. Child (premature) born 7.1.31; admitted to hospital and vaccinated successfully 13.1.31; did not develop smallpox.

Case 19.-Onset of mother's illness 21.11.32; rash 25.11.32. Child born 18.11.32; admitted to hospital and vaccinated successfully 26.11.32; did not develop smallpox.

B. BORN AT THE ONSET OF THE MOTHER'S ILLNESS.

Case 20.-Onset of mother's illness 14.5.29; rash 16.5.29. Child born 14.5.29; admitted to hospital and vaccinated successfully 18.5.29; did not develop smallpox.

Case 21.-Onset of mother's illness 23.10.29; rash ? 25.10.29. Child born (premature) 23.10.29; not vaccinated; rash 7.11.29; admitted to hospital 9.11.29; died 24.11.29.

Case 22.-Onset of mother's illness 14.11.29; rash 16.11.29. Child born 14.11.29; admitted to hospital and vaccinated successfully 21.11.29; rash 29.11.29; recovered.

Case 23. - Onset of mother's illness 28.4.31; rash 30.4.31. Child born 30.4.31; admitted to hospital and vaccinated successfully 5.5.31; rash 13.5.31; recovered.

Case 24.-Onset of mother's illness 22.6.31; rash 24.6.31. Child born 22.6.31; not vaccinated; rash 7.7.31; recovered.

It is to be observed that those infants who contracted smallpox subsequent to birth developed the focal rash fourteen days after the appearance of the mother's rash, and, further, that these cases either were not vaccinated or were vaccinated late in the incubation period. 
C. BORN DURING THE FIRST WEEK OF THE MOTILE'S RASH.

Case 25.-Onset of mother's illness 29.3.30; rash 31.3.30. Child (second of binovular twins) born in hospital and successfully vaccinated 6.4.30; did not develop smallpox. First twin (case 13), similarly vaccinated, developed smallpox on the eleventh day of extra-uterine life.

D. Born late in mother's illness (after the first week of her focal rash).

Case 26.-Mother's rash 14.10.29. Child born in hospital and successfully vaccinated 21.10.29; did not develop smallpox.

Case 27.-Mother's rash 25.11.29. Child born in hospital and successfully vaccinated 4.12.29; did not develon smallpox.

Case 28.-Mother successfully vaccinated 23.3.30; rash 25.3.30. Child born in hospital and successfully vaccinated 5.4.30; did not develop smallpox.

Case 29.-Mother's rash 12.4.31. Child born in hospital and successfully vaccinated 23.4.31; did not develon smallpox.

Case 30.-Mother's rash 17.7.30. Child born in hospital and successfully vaccinated 31.7.30; did not develop smallpox.

Case 31.-Mother's rash 9.6.30. Child born in hospital and successfully vaccinated 18.6.30; did not develop smallpox.

Case 32.-Mother's rash 24.4.30. Child born in hospital 15.5.30; vaccinated unsuccessfully 15.5.30; vaccinated successfully 20.5.30; did not develop smallpox. This child was fortunate to escape smallpox in that successful vaccination was delayed until the sixth day.

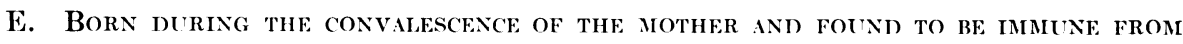
SMALLPOX.

Case 33.-Mother's rash 14.12.29. Child born in hospital 8.1.30; unsuccessfully vaccinated on 8, 10 and 12 January, 1930; did not develop smallpox.

Case 34.-Mother's rash 1.6.29. Twins born 21.7.29; each vaccinated unsuccessfully on three occasions; did not develop smallpox. A description of this case has been given elsewhere (Marsden"2).

\section{General discussion and conclusions.}

While clearly recognizing that any general inferences drawn from the small number of cases which constitute the present series would be unscientific and might even prove to be definitely misleading, we consider that the evidence afforded justifies certain particular conclusions.

In the first place our cases bear out the already well-recognized fact that when both mother and foetus are infected they do not pass through the disease simultaneously, except perhaps in rare instances. In other words, the foetus does not become infected synchronously with the mother but subsequently, and then the foetus passes through an individual period of incubation.

Secondly support is forthcoming for the theory that, as in the case of syphilis, it is, if not exactly fortuitous, more or less of an accident if infection is inherited. All the cases (seventeen) in groups 1, 2 and 3 were infected, whereas those in group 4 (seventeen) escaped. As far as can be seen these cases were at equal risk; with the exception of cases 18 and 19, and possibly of case 9 , all were in utero at the onset of the illness in the mother. 
Furthermore, it is difficult to explain on any other hypothesis the course of events in cases 13 and 25, binovular twins born on the seventh day of their mother's focal rash, one of whom, successfully vaccinated at birth, developed a smallpox eruption on the eleventh day of extra-uterine life, while the other, similarly treated, remained free from the disease. If, then, it be accepted that in the present state of our knowledge it is impossible to explain what factor, other than chance, determines whether a woman suffering from smallpox shall transmit the infection to her unborn foetus, the interesting question still remains whether the child is at equal risk throughout its mother's attack, or whether there is some stage of her illness, a point of particular danger to the infant, when infection of the foetus is most likely to occur.

As is well known, after infection with the virus of smallpox there follows a latent (incubation) period during which the virus apparently undergoes some proliferative process in the body of the host until, at about the twelfth day, the virus generalizes and symptoms of what is known as the toxaemic stage of smallpox become manifest. Paschen ${ }^{3}$ considers that this sudden inundation of the blood with virus shows that primarily one or more lesions giving enormous multiplication of virus must have been created, and that this lesion must lie in the lungs. He points out that, although nobody has yet seen this primary focus, the employment of neurolapine is followed by the production of gross pulmonary lesions. According to the embolic theory, formulated to explain the nature and production of the focal rash, within about twenty-four hours from the point when generalization commences, the blood stream is flooded with infective particles, which, subsequently becoming lodged in the minute capillaries of the skin and certain mucous membrances, give rise to the specific focal lesions. The eruption is known to be determined to certain areas of the body surfaces which, in virtue of their susceptibility to vascular variations, are peculiarly favourable sites for the deposition of the particulate virus, although why this process should be limited to the skin and adjacent mucous membrances is not properly understood.

On general grounds the most likely time for the foetus to become infected would appear to be during the mother's ' septicaemic' stage, immediately following the onset of her illness. If this be the true method of transference of infection, via the maternal blood stream, we are quite unable to explain why it does not always occur, but we consider that our evidence supports the view. In the vast majority of instances smallpox infection is acquired by way of the respiratory tract and about fourteen days afterwards (sometimes as late as seventeen days and more rarely as early as twelve), the outcrop of the focal rash commences. But however inherited infection is acquired before birth it is plain that it cannot be by way of the respiratory tract, and it is natural to look for some modification of the usual incubation period in accordance with the unusual channel of infection.

Inoculation with smallpox material, variolation of the human subject, was a fairly common procedure in pre-Jennerian times, and records are 
available of the course of the disease produced in this manner. Following the appearance on the fourth day at the inoculation site of a local lesion which progressed in a manner similar to that which is adopted by vaccinia, a general focal rash made its appearance usually on the eleventh day. In a case of accidentally inoculated smallpox recently under the observation of one of us the first signs of a general eruption were noted on a day calculated, from evidence supplied by the local lesion, to be the twelfth following inoculation.

It may be that herein lies the clue which we have been seeking and study of the details of cases 1 to 11 reveals a period, between the onset of the mother's illness and the appearance of the focal rash on the child, which closely corresponds to the incubation period of inoculated smallpox. The cases in group 3, all with the exception of number 13 born synchronously with the outcrop of the mother's focal rash, if regarded on the reasonable assumption that they were infected at or about the time of separation (birth) present a similar incubation period. This assumption might legitimately be challenged on the grounds that, as the interval between the onset of the mother's illness and the appearance of the child's rash in the cases which form this group varies from fourteen to eighteen days, it would be equally justifiable to conclude that infection of the foetus had taken place at the commencement of the mother's illness and then had been succeeded by a normal incubation period. We would point out, however, that such a postulate could not be extended to include the cases in groups 1 and 2 .

We conclude, therefore, that our evidence suggests that the bulk of foetal infections are acquired in utero at the time of the mother's ' septicaemia' by a process the results of which, particularly in regard to length of incubation period, are akin to those of inoculation (cases in groups 1 and 2). But that if the foetus then escapes it may similarly acquire infection at or about the time of separation from its parent (cases in group 3 and possibly also those in group 2), and that this is particularly liable to occur if the mother's rash is in its early stages when the birth takes place. That if the child escapes these two contingencies it escapes congenital infection; but that generally, and in the absence of prompt successful vaccination, the disease, acquired by the more usual respiratory route, will show itself after the normal period of incubation (cases 21 to 24). And lastly, there is evidence that if the foetus escapes intra-uterine infection and remains in utero until the mother is convalescent from her attack of smallpox, it may then be born immune, at any rate temporarily, from the disease (cases 33 and 34). It is realized, however, that these patients may have survived in utero an attack of smallpox of which they presented no superficial evidence at birth.

\section{REFERENCES.}

1. Schamberg, J. F., \& Kolmer, J. A., Acute Infectious Diseases, Second Edit., Philadelphia, 1928, 158.

2. Marsden, J. P., Brit. J. Child. Dis., London, 1930, XXVII, 183.

3. Paschen, E., Brit. Med. J., London, 1932, ii, 957. 